\title{
Unstimulated Saliva-Related Caries Risk Factors in Individuals with Cystic Fibrosis: A Cross- Sectional Analysis of Unstimulated Salivary Flow, pH, and Buffering Capacity
}

\author{
Alaa A. Alkhateeb ${ }^{a}$ Lloyd A. Mancl ${ }^{a}$ Richard B. Presland ${ }^{a, b}$ \\ Marilynn L. Rothen ${ }^{\mathrm{a}}$ Donald L. Chi ${ }^{\mathrm{a}}$ \\ a Department of Oral Health Sciences, School of Dentistry, and ${ }^{\mathrm{b}}$ Division of Dermatology, School of Medicine, \\ University of Washington, Seattle, Wash., USA
}

\section{Key Words}

Buffering capacity $\cdot$ Cystic fibrosis · Dental caries $\cdot \mathrm{pH}$.

Salivary flow $\cdot$ Unstimulated saliva

\begin{abstract}
Salivary flow rate, $\mathrm{pH}$, and buffering capacity are associated with dental caries, but studies from the cystic fibrosis (CF) literature are inconclusive regarding these salivary factors and caries. The aim of this study was to evaluate these factors and their associations with dental caries in individuals with CF. Unstimulated whole saliva was collected from individuals aged 6-20 years at Seattle Children's Hospital CF Clinic, USA $(n=83)$. Salivary flow rate was measured in milliliters per minute. Salivary $\mathrm{pH}$ was assessed using a laboratory $\mathrm{pH}$ meter. Buffering capacity was assessed by titration with $\mathrm{HCl}$. The outcome measure was caries prevalence, defined as the number of decayed, missing, or filled primary and permanent tooth surfaces. Spearman's rank correlation coefficient and the $t$ test were used to test for bivariate associations. Multiple variable linear regression models were used to (1) run confounder-adjusted analyses and (2) assess for potential interactions. There was no significant association between salivary flow rate or buffering capacity and caries
\end{abstract}

() 2016 S. Karger AG, Basel

E-Mail karger@karger.com www.karger.com/cre prevalence. There was a significant negative association between salivary $\mathrm{pH}$ and caries prevalence, but this association was no longer significant after adjusting for age. There was no significant interaction between salivary flow rate and buffering capacity or between antibiotic use and the 3 salivary factors. Our results indicate that unstimulated salivary factors are not associated with dental caries prevalence in individuals with CF. Future studies should investigate other potential saliva-related caries risk factors in individuals with CF such as cariogenic bacteria levels, salivary host defense peptide levels, and medication use. @ $2016 \mathrm{~S}$. Karger AG, Basel

Cystic fibrosis (CF) is an autosomal recessive disease that results in abnormal transport of chloride and sodium across the cell membrane. This abnormality affects hydration and mucociliary transport within exocrine glands, including the salivary glands [Aps et al., 2002; Rowe et al., 2005]. While low salivary flow rates, acidic $\mathrm{pH}$, and low buffering capacity are known risk factors for caries [Dodds et al., 2005], the literature is inconsistent on whether these salivary factors are associated with caries in individuals with CF. One study showed increased salivary 
flow rates in individuals with CF compared to controls [Barbero and Chernick, 1958], while other studies report decreased salivary flow rates [Wiesmann et al., 1972; Davies et al., 1990], and a recent study showed no significant difference [Peker et al., 2014]. As with the salivary flow rates, the literature is inconsistent regarding salivary $\mathrm{pH}$ levels in individuals with CF [Chernick et al., 1961; Chauncey et al., 1962; Boat et al., 1974; Aps et al., 2002; Catalan et al., 2011; Peker et al., 2015]. A recent study found no significant differences in salivary buffering capacity levels between individuals with CF and controls [Peker et al., 2014].

Many of the preceding studies are older and have less relevance today because CF medications and treatments have changed significantly in recent years [Chi, 2013]. The last large-scale oral health study on individuals with CF in the USA was published in 1980 [Primosch, 1980]. Newer knowledge based on up-to-date data is needed to help understand caries risk and guide the development of clinical protocols and interventions aimed at protecting the health and well-being of individuals with CF. To better understand how salivary characteristics are related to caries within a population of US individuals with CF, we tested 5 hypotheses:

- A higher unstimulated salivary flow rate is associated with lower caries prevalence

- Elevated unstimulated salivary pH (i.e. more basic saliva) is associated with lower caries prevalence

- Elevated unstimulated salivary buffering capacity is associated with lower caries prevalence

- There is an interaction between the unstimulated salivary flow rate and buffering capacity

- There are interactions between the 3 salivary factors and antibiotic use

\section{Materials and Methods}

\section{Study Design and Participants}

This was a cross-sectional study consisting of a convenience sample of participants with CF aged 6-20 years. We recruited individuals presenting for outpatient or inpatient medical care at Seattle Children's Hospital Cystic Fibrosis Clinic from March 2014 to December 2015. The recruitment goal was to enroll as many eligible participants as possible during this period. We approached 88 eligible individuals with CF and enrolled 83 participants. Individuals who did not participate had other medical appointments or studies to attend. Written informed consent was obtained from the parent or caregiver of child participants or directly from adult participants. The study was approved by the Seattle Children's Hospital Institutional Review Board (study No. 14894).
Saliva Collection, Processing, and Measures

Unstimulated whole saliva samples were collected using a previously published protocol used to successfully collect saliva samples from children with special health care needs as young as 6 years of age [Lundgren et al., 1996; Devic et al., 2014]. Immediately prior to saliva collection, participants were asked to swallow the saliva present in the mouth. Participants were then asked to expectorate into a sterile $50-\mathrm{ml}$ tube once per minute for $15 \mathrm{~min}$. After collection, saliva samples were placed on ice and transported to the University of Washington for immediate processing and analysis.

For salivary flow measurements, saliva samples were weighed on a top-pan balance to determine the amount of saliva collected. Salivary flow rate was calculated for each sample in grams per minute (equivalent to milliliters per minute) [Dawes, 2008].

For salivary $\mathrm{pH}$ assessment, samples were centrifuged for 2 $\min$ at $10,000 \mathrm{~g}$ to remove cell debris. Resting salivary $\mathrm{pH}$ was determined with $1 \mathrm{ml}$ of saliva using a laboratory $\mathrm{pH}$ meter (Orion 320; Thermo Electron Corporation), accurate to $0.02 \mathrm{pH}$ units in aqueous buffers. $\mathrm{pH}$ was measured at room temperature (about $20^{\circ} \mathrm{C}$ ), which is consistent with the Ericsson method and previously published protocols [Ericsson, 1959; Kitasako et al., 2008]. To control for the effects of ambient temperature on $\mathrm{pH}$ measurements, the $\mathrm{pH}$ meter was calibrated at room temperature.

Buffering capacity was determined based on methods developed by Ericsson [1959]. Saliva (either 0.5 or $1 \mathrm{ml}$, depending on the amount available) was mixed with $5 \mathrm{~mm} \mathrm{HCl}(1.5$ or $3 \mathrm{ml}$, respectively) in a $10-\mathrm{ml}$ polypropylene tube and mixed vigorously. Buffering capacity was measured after a 10 -min incubation period. Samples were categorized based on the final pH [Ericsson, 1959]:

- High buffering capacity: final $\mathrm{pH}>4.75$

- Medium buffering capacity: final $\mathrm{pH}=4.25-4.75$

- Low buffering capacity: final $\mathrm{pH}<4.25$

\section{Outcome Measure}

The outcome measure was dental caries prevalence (or total caries experience). Dental examinations were completed after saliva collection by trained and calibrated pediatric dentists or a dental hygienist. The intrarater reliability of the examiners was good $($ kappa coefficient $=0.97)$. Participants were asked to sit in a reclining chair in a private medical examination room. A head lamp was used to provide additional lighting and maximize the visualization of the teeth. Caries prevalence was measured by visual inspection of the teeth after brushing all tooth surfaces with a dry toothbrush. Each primary and permanent tooth surface was classified as decayed, filled, or missing using adapted NIDCR Early Childhood Caries Collaborative Centers Criteria [Warren et al., 2009], which are based on the WHO criteria. We defined caries prevalence in 3 ways: total number of decayed, filled, and missing primary and permanent tooth surfaces (dmfs + DMFS), total number of decayed, filled, and missing surfaces plus the presence of noncavitated white spot lesions (dmfsw + DMFSW), and dmfsw + DMFSW as a proportion of the total number of tooth surfaces present in the mouth.

\section{Additional Study Variables}

Four additional study variables were collected via a written survey instrument. Age, modeled as a continuous variable, was conceptualized as a potential confounder based on previous 
Table 1. Bivariate associations between study variables

\begin{tabular}{lllll}
\hline & $\begin{array}{l}\text { Unstimulated } \\
\text { salivary flow rate, } \\
\text { ml/min }\end{array}$ & $\begin{array}{l}\text { Unstimulated } \\
\text { salivary pH }\end{array}$ & $\begin{array}{l}\text { Unstimulated } \\
\text { salivary buffer- } \\
\text { ing capacity }\end{array}$ & $\begin{array}{l}\text { Dental caries } \\
\text { prevalence }\end{array}$ \\
\hline $\begin{array}{l}\text { Unstimulated salivary flow rate } \\
\text { Unstimulated salivary pH }^{\mathrm{a}}\end{array}$ & - & $0.23^{*}$ & 0.06 & 0.04 \\
Unstimulated salivary buffering capacity $^{\mathrm{a}}$ & - & - & $0.40^{* *}$ & $-0.25^{*}$ \\
Age $^{\mathrm{a}}$ & - & - & - & -0.07 \\
Antibiotic use $^{\mathrm{b}}$ & 0.08 & $-0.50^{* *}$ & -0.19 & $0.35^{* *}$ \\
$\quad \begin{array}{l}\text { No } \\
\text { Yes }\end{array}$ & 0.26 & 7.1 & 4.2 & 1.86 \\
\hline
\end{tabular}

Dental caries prevalence $=$ Total number of decayed, missing, or filled primary and permanent tooth surfaces (dmfs + DMFS). ${ }^{\text {a }}$ The significance of Spearman's rank correlation coefficient is reported using the following convention: ${ }^{*} \mathrm{p}<0.05,{ }^{* *} \mathrm{p}<0.01{ }^{\mathrm{b}}$ Indicates means. None of the relationships between antibiotic use and variables were statistically significant from the t test.

work indicating significant associations with salivary factors and caries [Nassar et al., 2014; Dye et al., 2007]. We collected data on sex (male or female), public health insurance (no or yes), and whether participants had used any antibiotics within the previous 2 months (no or yes). Antibiotic use was modeled as a potential effect modifier of the 3 salivary factors. A fifth variable, oral hygiene status, was collected prior to the dental examination. To assess oral hygiene status, we used the Plaque Index System developed by Löe [1967] based on the amount of plaque that could be removed from tooth surfaces with a periodontal probe. Oral hygiene was classified as good if there was no plaque present or the amount of plaque removed with the probe was minimal, fair if the amount of plaque removed was moderate, or poor if there was an abundance of plaque.

\section{Data Analyses}

Univariate statistics were generated to describe the study population. Spearman's rank correlation coefficient was used to assess the bivariate associations between (1) the 3 salivary factors (flow rate, $\mathrm{pH}$, and buffering capacity), (2) age and the 3 salivary factors, (3) the 3 saliva factors and dental caries prevalence, and (4) age and dental caries prevalence [Dye et al., 2007; Nassar et al., 2014]. The $t$ test was used to assess the relationships between antibiotic use and (1) the 3 salivary factors and (2) dental caries. We used twosided tests $(\alpha=0.05)$.

Multiple variable linear regression models were used to test for associations between salivary factors that were significantly associated with caries in the bivariate analyses, adjusting for any confounders, and to test for the interactions between (1) salivary flow rate and buffering capacity and (2) the 3 salivary factors and antibiotic use. For all regression models, salivary measures and caries prevalence were transformed using the square root to normalize the distribution. Because there were no differences in results based on the 3 definitions of caries prevalence, all findings were reported using the dmfs + DMFS definition. All analyses were completed using the Statistical Package for Social Sciences 18.0 for Windows.

Salivary Factors and Caries in Cystic Fibrosis

\section{Results}

\section{Descriptive Statistics}

The mean age of the study participants was 11.9 years (standard deviation, SD: 4.0 years; median age: 11.0 years). Most participants were female (54.3\%). About 1 in 3 were publicly insured. In terms of oral hygiene status, $25.3 \%$ had good hygiene, $31.3 \%$ had fair hygiene, $26.5 \%$ had poor hygiene, and $16.9 \%$ had missing hygiene data. Sixty-five percent of study participants reported using antibiotics.

Five participants, aged 8-13, were unable to provide the minimum volume required for salivary analyses $(0.5$ $\mathrm{ml}$ ) and were excluded from the salivary analyses. The mean $( \pm \mathrm{SD})$ salivary flow rate was $0.28 \pm 0.22 \mathrm{ml} / \mathrm{min}$. The mean salivary $\mathrm{pH}$ was $7.14 \pm 0.48$. The mean salivary buffering capacity was $4.26 \pm 0.86$. Of the study population, $26 \%$ had high salivary buffering capacity (final $\mathrm{pH}$ $>4.75$ ), $26 \%$ had medium buffering (final $\mathrm{pH}=4.25-$ 4.75 ), and $48.1 \%$ had low buffering capacity (final $\mathrm{pH}$ $<4.25)$. The mean caries prevalence (dmfs + DMFS) was $2.60 \pm 4.35$, with a range of $0-21$ tooth surfaces affected by caries.

\section{Bivariate Associations}

Table 1 presents the associations between study variables. There was a significant positive association between salivary $\mathrm{pH}$ and flow rate $(\mathrm{r}=0.23)$, and between salivary $\mathrm{pH}$ and buffering capacity $(\mathrm{r}=0.40)$. There was a positive association between salivary flow rate and buffering capacity $(r=0.06)$, but the association was not statistically significant. Age was significantly associated with 
salivary $\mathrm{pH}(\mathrm{r}=-0.50)$ but not with salivary flow rate or buffering capacity.

There was a significant negative association between salivary $\mathrm{pH}$ and caries prevalence $(\mathrm{r}=-0.25)$. There was no significant association between flow rate and caries prevalence or between buffering capacity and caries prevalence. Age was significantly and positively associated with caries $(r=0.35)$. Antibiotic use was not significantly associated with any of the 3 salivary factors or with dental caries.

Based on the bivariate analyses, only salivary $\mathrm{pH}$ was significantly associated with caries, and age confounded this relationship. The linear regression model indicated no association between salivary $\mathrm{pH}$ and dental caries prevalence after adjusting for age $(\beta=-0.015 ; 95 \% \mathrm{CI}=$ -3.72 to $3.32 ; \mathrm{p}=0.91$ ). In subgroup analyses, the association between salivary $\mathrm{pH}$ and caries prevalence failed to reach statistical significance when evaluated separately for children aged $6-11$ years $(p=0.91)$, adolescents aged $12-17$ years $(\mathrm{p}=0.24)$, or adults aged $18-20$ years $(\mathrm{p}=0.38)$.

There was no significant interaction between salivary buffering capacity and flow rate $\left(\beta_{\text {interaction }}=-0.70 ; 95 \%\right.$ $\mathrm{CI}=-1.78$ to $0.99 ; \mathrm{p}=0.56$ ), nor were there significant interactions present between antibiotic use and salivary flow $(\mathrm{p}=0.10), \mathrm{pH}(\mathrm{p}=0.61)$, or buffering capacity $(\mathrm{p}=$ $0.90)$.

\section{Discussion}

This is the largest US clinical oral health study published since 1980 on caries in individuals with CF. We focused on 3 unstimulated salivary measures - salivary flow, $\mathrm{pH}$, and buffering capacity. Our data indicate that none of these salivary factors were significantly associated with dental caries in a population of individuals with CF aged 6-20 years. In addition, there was no significant interaction between salivary flow rate and buffering capacity, nor were there significant interactions between antibiotic use and the 3 salivary factors.

That unstimulated salivary flow rate was not associated with caries prevalence is consistent with practicebased findings for US children aged 9-17 years [CunhaCruz et al., 2013] and with findings in healthy children aged 7-15 years in India [Pandey et al., 2015]. In the present study, the mean \pm SD unstimulated salivary flow rate for children aged 6-12 years was $0.30 \pm 0.25 \mathrm{ml} / \mathrm{min}$, which is less than the mean unstimulated salivary flow rate of $0.53 \pm 0.30 \mathrm{ml} / \mathrm{min}$ found in Turkish children with
CF aged 3-12 years [Peker et al., 2015]. A potential explanation is based on previous work indicating that caries protection is associated with stimulated saliva, rather than unstimulated saliva, because stimulated saliva contains higher mineral content and leads to greater buffering capacity and salivary clearance [Edgar et al., 1994; Lagerlof and Oliveby, 1994]. This suggests that the specific attributes of saliva are more important than saliva quantity in caries prevention. Future research should verify whether flow rates in our study are typical for individuals with CF and examine the relationship between stimulated salivary flow rate and caries in individuals with CF.

We also found that unstimulated salivary $\mathrm{pH}$ was not associated with dental caries prevalence after adjusting for age. This is consistent with findings from one pediatric study [Singh et al., 2015], but inconsistent with 2 other studies focusing on children [Cunha-Cruz et al., 2013; Pandey et al., 2015]. One reason for the discrepancy regarding salivary $\mathrm{pH}$ and caries prevalence is that studies typically measure $\mathrm{pH}$ once, which does not capture the dynamic relationship between $\mathrm{pH}$ and demineralization. Study protocols involving salivary $\mathrm{pH}$ collection need to be standardized to ensure comparability across studies. Even with nonstandardized protocols, however, in the present study, the mean unstimulated salivary $\mathrm{pH}$ for adolescents aged $11-15$ years was $7.14 \pm 0.42$, which is similar to the mean $\mathrm{pH}$ for Turkish children with CF aged 11-15 years $(7.55 \pm 0.61)$ [Peker et al., 2015]. Another observation in the present study is that salivary $\mathrm{pH}$ became increasingly acidic with older age, which is consistent with previous findings for non-CF populations [Cunha-Cruz et al., 2013; Pandey et al., 2015]. The reason for potential age-related changes in salivary $\mathrm{pH}$ for individuals with $\mathrm{CF}$ is unknown. Biological or behavioral mechanisms could explain these age-related changes during childhood and adolescence. Future work should examine the longitudinal relationship between $\mathrm{pH}$ and caries and examine reasons why unstimulated salivary $\mathrm{pH}$ appears to become more acidic with age.

In addition, unstimulated salivary buffering capacity was not associated with caries in our study. The association between salivary buffering capacity and dental caries is inconsistent based on previous studies. One publication reported a weak negative association between unstimulated salivary buffering capacity and caries in healthy children aged 7-14 years [Prabhakar et al., 2009], whereas another study reported a positive association between stimulated salivary buffering capacity and caries in children aged 9-17 years [Cunha-Cruz et al., 2013]. There are 2 possible explanations for these inconsistencies. The 
first is the different types of saliva to measure buffering capacity (stimulated vs. unstimulated). The second is the method used to measure buffering capacity. Some studies, including our analyses, used the Ericsson method and pH meters [Prabhakar et al., 2009], which is considered the gold standard because of its objectivity, whereas other studies use $\mathrm{pH}$ colorimetric strips [Cunha-Cruz et al., 2013] that may introduce bias because of rater subjectivity. Future research should adopt gold standard laboratory methods in measuring buffering capacity and interpret findings carefully based on the type of saliva collected for the study.

Finally, we found that the association between salivary buffering capacity and caries was not moderated by flow rate. To date, no studies have examined this interaction, despite evidence that both salivary factors are important in caries initiation and progression [Leone and Oppenheim, 2001]. Given the complexity of salivary factors, future research involving larger study populations should continue to examine how various salivary factors interact in the caries process. In addition, there were no significant interactions between antibiotic use and the 3 salivary factors. One limitation of a binary antibiotic measure is that it does not capture the specific type, dose, or frequency of antibiotic use that might be important correlates of salivary characteristics and caries. For instance, mutans streptococci, the main bacteria implicated in dental caries, are Gram-positive and would be expected to be targeted by antibiotics like penicillin. Future analyses should examine the specific types, dose, and frequency of antibiotics associated with CF therapies that could protect against caries.

In terms of external generalizability of study findings, there are no direct studies focusing on individuals with $\mathrm{CF}$ to which an assessment can be made. However, our observed caries rates are consistent with conclusions from a recent systematic review, which reported that children with CF have lower caries rates than children without $\mathrm{CF}$ whereas adolescents have higher caries rates than adolescents without CF [Chi, 2013]. In the present study, the mean of decayed and filled surfaces of primary teeth (dfs) for children with CF aged 6-11 was 0.69, which was lower than the US dfs mean of 3.6 for children aged 2-11 years [Dye et al., 2007]. In our study, the mean of decayed, filled, and missing surfaces of permanent teeth (DMFS) for adolescents with CF aged 12-19 was 3.60, which is higher than the US DMFS mean of 1.03 for adolescents aged 12-19 years [Dye et al., 2007]. Furthermore, $92.8 \%$ of our study participants were white, which is slightly higher but consistent with the US CF preva- lence rate of $85 \%$ [Cystic Fibrosis Foundation, 2016]. These indirect comparisons suggest that our study findings are likely to be generalizable to US individuals with CF aged 6-20 years, but additional multicenter studies would help to improve generalizability for future investigations.

This study had several limitations. First, the study population was recruited from a single center. Future studies should investigate saliva parameters as caries risk factors from multiple sites. Second, all participants were medical care utilizers. As such, the study was likely to have been biased toward healthier individuals with CF and cannot be generalized to nonutilizers of medical care. Future research should seek to identify and recruit individuals with CF who may be less connected to the medical care system, although we acknowledge that recruiting nonutilizers can be a challenge. Third, $55.4 \%$ of the study population had private insurance, which may further bias our study population toward higher-income individuals with lower caries rates. However, based on data from the Cystic Fibrosis Foundation Registry, this rate is consistent with private insurance rates from the broader US CF population [Cystic Fibrosis Foundation, 2016]. Future studies should make concerted efforts to enroll low-income individuals with CF. Fourth, our model was incomplete, and the study was cross-sectional. Future studies should further investigate additional salivary factors like oral bacteria levels and expression levels of host defense peptides as well as factors that may affect salivary expression and quality like the use of medications, including specific antibiotics. In addition, it is possible that variation in CF genotype mutations could further explain differences in salivary characteristics within a population of individuals with CF [Aps et al., 2002] and should be examined in future research. This limitation highlights the need to conduct longitudinal studies to examine how changes in salivary factors influence caries rates over time.

In conclusion, we found that unstimulated salivary flow rate, $\mathrm{pH}$, and buffering capacity were not associated with caries in our population of individuals with CF recruited from a children's hospital CF clinic. There is a need for larger, multicenter, longitudinal studies to better understand how salivary and other social, behavioral factors, medical, and biological factors influence caries initiation and progression in individuals with CF. These data can then be used to update dental treatment protocols for patients with CF and develop interventions aimed at protecting the oral and systemic health of these medically vulnerable individuals. 


\section{Acknowledgments}

We would like to thank the participating individuals and families who made this study possible. Thank you to the staff at Seattle Children's Hospital CF Clinic and the Pediatric Clinical Research Center for help in recruiting and scheduling participants and the University of Washington School of Dentistry Regional Clinical Dental Research Center for study supplies. Funding for this study was provided by the US National Institute of Dental and Craniofacial Research (grant No. K08DE020856 and P30DK089507), the University of Washington Institute of Translational Health Sciences (supported by the National Center for Advancing Translational Sciences of the National Institutes of Health under grant No. UL1TR000423), the SunStar Americas Corporation, the Cystic Fibrosis Foundation, and the Center for Advanced Study in the Behavioral Sciences (CASBS) at Stanford University. The funders had no role in the study design, data collection and analysis, decision to publish, or preparation of the manuscript.

\section{Disclosure Statement}

The authors have no conflicts of interests to declare.

\section{Author Contributions}

Designed the study: D.L.C. Performed the dental examination: D.L.C. and M.L.R. Performed the saliva analysis: A.A.A. and R.B.P. Analyzed the data: A.A.A., L.A.M., and D.L.C. Wrote the manuscript: A.A.A., L.A.M., M.L.R., R.B.P., and D.L.C.

\section{References}

Aps JK, Delanghe J, Martens LC: Salivary electrolyte concentrations are associated with cystic fibrosis transmembrane regulator genotypes. Clin Chem Lab Med 2002;40:345-350.

Barbero GJ, Chernick W: Function of the salivary gland in cystic fibrosis of the pancreas. Pediatrics 1958;22:945-952.

Boat TF, Wiesman UN, Pallavicini JC: Purification and properties of the calcium-precipitable protein in submaxillary saliva of normal and cystic fibrosis subjects. Pediatr Res 1974;8:531-539.

Catalan MA, Scott-Anne K, Klein MI, Koo H, Bowen WH, Melvin JE: Elevated incidence of dental caries in a mouse model of cystic fibrosis. PLoS One 2011;6:e16549.

Chauncey HH, Levine DM, Kass G, Shwachman $\mathrm{H}$, Henriques BL, Kulczyeki LL: Composition of human saliva. Parotid gland secretory rate and electrolyte concentration in children with cystic fibrosis. Arch Oral Biol 1962;7:707-713.

Chernick WS, Barbero GJ, Parkins FM: Studies on submaxillary saliva in cystic fibrosis. J Pediatr 1961;59:890-898.

Chi DL: Dental caries prevalence in children and adolescents with cystic fibrosis: a qualitative systematic review and recommendations for future research. Int J Paediatr Dent 2013;23: 376-386.

Cunha-Cruz J, Scott J, Rothen M, Mancl L, Lawhorn T, Brossel K, Berg J; Northwest Practice-based RCiE-bD: salivary characteristics and dental caries: evidence from general dental practices. J Am Dent Assoc 2013;144:e31-e40.

Cystic Fibrosis Foundation: Cystic Fibrosis Foundation Patient Registry 2014 Annual Data Report. Bethesda, Cystic Fibrosis Foundation, 2016.

Davies H, Bagg J, Goodchild MC, McPherson MA: Examination of submandibular fluid in cystic fibrosis. Acta Univ Carol Med (Praha) 1990;36:84-85.

Dawes C: Salivary flow patterns and the health of hard and soft oral tissues. J Am Dent Assoc 2008;139(suppl):18S-24S.
Devic I, Shi M, Schubert MM, Lloid M, Izutsu KT, Pan C, Missaghi M, Morton TH, Mancl LA, Zhang J, Presland RB: Proteomic analysis of saliva from patients with oral chronic graftversus-host disease. Biol Blood Marrow Transplant 2014;20:1048-1055.

Dodds MW, Johnson DA, Yeh CK: Health benefits of saliva: a review. J Dent 2005;33:223233.

Dye BA, Tan S, Smith V, Lewis BG, Barker LK, Thornton-Evans G, Eke PI, Beltran-Aguilar ED, Horowitz AM, Li CH: Trends in oral health status: United States, 1988-1994 and 1999-2004. Vital Health Stat 11 2007;248:192.

Edgar WM, Higham SM, Manning RH: Saliva stimulation and caries prevention. Adv Dent Res 1994;8:239-245.

Ericsson Y: Clinical investigation of the salivary buffering action. Acta Odontol Scand 1959; 17:131-165.

Kitasako Y, Burrow MF, Stacey M, Huq L, Reynolds EC, Tagami J: Comparative analysis of three commercial saliva testing kits with a standard saliva buffering test. Aust Dent J 2008;53:140-144.

Lagerlof F, Oliveby A: Caries-protective factors in saliva. Adv Dent Res 1994;8:229-238.

Leone CW, Oppenheim FG: Physical and chemical aspects of saliva as indicators of risk for dental caries in humans. J Dent Educ 2001;65: 1054-1062.

Löe H: The gingival index, the plaque index, and the retention index systems. J Periodontol 1967;60,159-162.

Lundgren T, Twetman S, Johansson I, Crossner CG, Birkhed D: Saliva composition in children and young adults with Papillon-Lefevre syndrome. J Clin Periodontol 1996;23:10681072.

Nassar M, Hiraishi N, Islam M, Otsuki M, Tagami J: Age-related changes in salivary biomarkers. J Dent Sci 2014;9:85-90.
Pandey P, Reddy NV, Rao VA, Saxena A, Chaudhary $\mathrm{CP}$ : Estimation of salivary flow rate, $\mathrm{pH}$, buffer capacity, calcium, total protein content and total antioxidant capacity in relation to dental caries severity, age and gender. Contemp Clin Dent 2015;6:S65-S71.

Peker S, Kargul B, Tanboga I, Tunali-Akbay T, Yarat A, Karakoc F, Ersu R, Dagli E: Oral health and related factors in a group of children with cystic fibrosis in Istanbul, Turkey. Niger J Clin Pract 2015;18:56-60.

Peker S, Mete S, Gokdemir Y, Karadag B, Kargul B: Related factors of dental caries and molar incisor hypomineralisation in a group of children with cystic fibrosis. Eur Arch Paediatr Dent 2014; 15:275-280.

Prabhakar A, Dodawad R, Os R: Evaluation of flow rate, $\mathrm{pH}$, buffering capacity, calcium, total protein and total antioxidant levels of saliva in caries free and caries active children an in vivo study. Int J Clin Pediatr Dent 2009; 2:9-12.

Primosch RE: Tetracycline discoloration, enamel defects, and dental caries in patients with cystic fibrosis. Oral Surg Oral Med Oral Pathol 1980;50:301-308.

Rowe SM, Miller S, Sorscher EJ: Cystic fibrosis. N Engl J Med 2005;352:1992-2001.

Singh S, Sharma A, Sood PB, Sood A, Zaidi I, Sinha A: Saliva as a prediction tool for dental caries: an in vivo study. J Oral Biol Craniofac Res 2015;5:59-64.

Warren JJ, Weber-Gasparoni K, Marshall TA, Drake DR, Dehkordi-Vakil F, Dawson DV, Tharp KM: A longitudinal study of dental caries risk among very young low SES children. Community Dent Oral Epidemiol 2009;37: 116-122.

Wiesmann UN, Boat TF, Di Sant'Agnese PA: Flow-rates and electrolytes in minor- salivary-gland saliva in normal subjects and patients with cystic fibrosis. Lancet 1972;ii:510512 . 